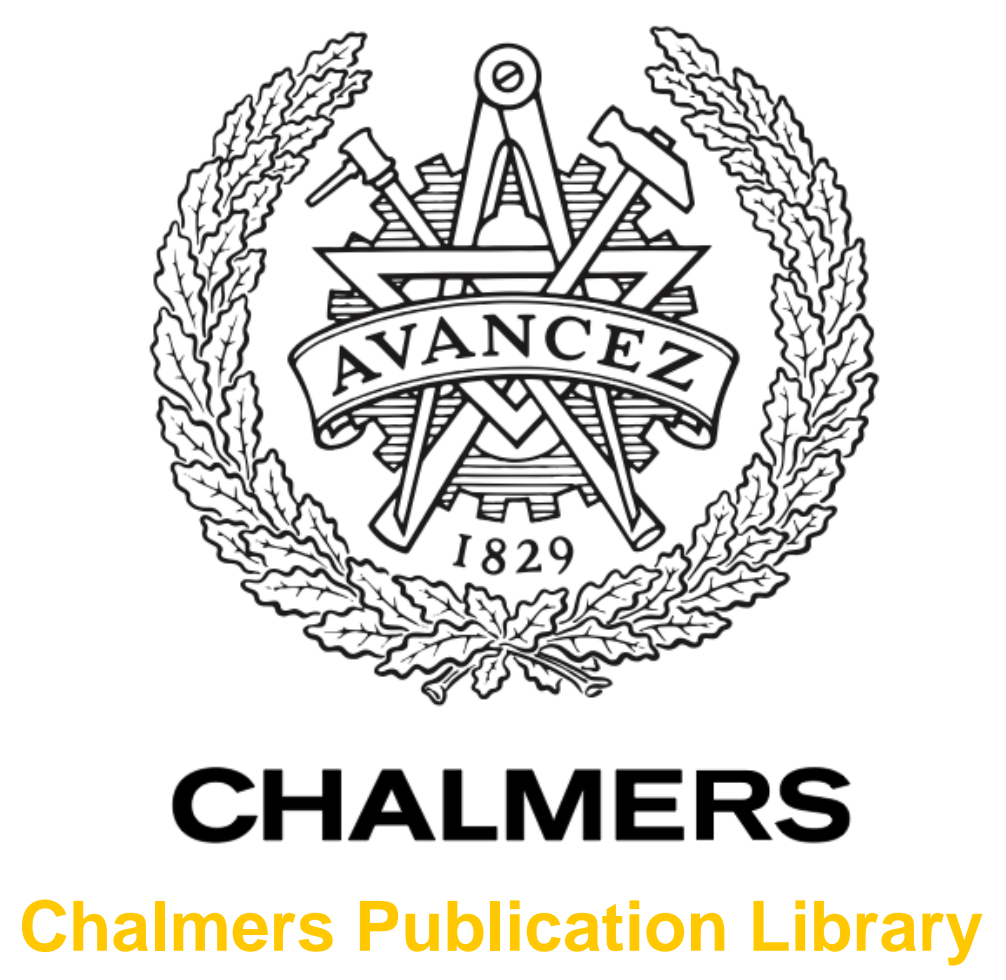

\title{
Evaluation of Classifier Topologies for the Real-time Classification of Simultaneous Limb motions
}

This document has been downloaded from Chalmers Publication Library $(\mathrm{CPL})$. It is the author's version of a work that was accepted for publication in:

in Proceedings of the 35th Annual International Conference of the IEEE Engineering in Medicine and Biology Society. Osaka, Jul 3-7, 2013.

Citation for the published paper:

Ortiz-Catalan, M. ; Brånemark, R. ; Håkansson, B. (2013) "Evaluation of Classifier Topologies for the Real-time Classification of Simultaneous Limb motions". in Proceedings of the 35th Annual International Conference of the IEEE Engineering in Medicine and Biology Society. Osaka, Jul 3-7, 2013.

Downloaded from: http://publications.lib.chalmers.se/publication/176133

Notice: Changes introduced as a result of publishing processes such as copy-editing and formatting may not be reflected in this document. For a definitive version of this work, please refer to the published source. Please note that access to the published version might require a subscription. 


\title{
Evaluation of Classifier Topologies for the Real-time Classification of Simultaneous Limb Motions
}

\author{
Max Ortiz-Catalan, Rickard Brånemark, and Bo Håkansson
}

\begin{abstract}
The prediction of motion intent through the decoding of myoelectric signals has the potential to improve the functionally of limb prostheses. Considerable research on individual motion classifiers has been done to exploit this idea. A drawback with the individual prediction approach, however, is its limitation to serial control, which is slow, cumbersome, and unnatural. In this work, different classifier topologies suitable for the decoding of mixed classes, and thus capable of predicting simultaneous motions, were investigated in real-time. These topologies resulted in higher offline accuracies than previously achieved, but more importantly, positive indications of their suitability for real-time systems were found. Furthermore, in order to facilitate further development, benchmarking, and cooperation, the algorithms and data generated in this study are freely available as part of BioPatRec, an open source framework for the development of advanced prosthetic control strategies.
\end{abstract}

\section{INTRODUCTION}

The simultaneous control of different degrees of freedom (DoF) is a highly desirable feature for a natural control of artificial limbs. Herein we refereed to "natural" as producing control analogous to that of an intact physiological system, which implies coordinated and simultaneous movements of more than one DoF. Furthermore, the input signals must be physiologically appropriate, and the feedback must be perceived as originating in the missing limb without requiring burdensome levels of concentration. This work is devoted to the decoding of mixed myoelectric signals (MES) in order to allow a simultaneous control of artificial limbs.

Mixed MES have been previously decoded using an array of 22 surface electrodes with offline accuracies up to $75 \%$ for 3 DoF [1]. Yatsenko et al. employed an algorithm based on principal component analysis, whitening, and orthonormalization of the feature vectors assuming linear relationships in the mixed MES. Based on the same principle, Jiang et al. proposed the biologically inspired Nonnegative Matrix Factorization (NMF) algorithm [2]. The NMF was tested for wrist movements satisfactorily predicting 2 out of $3 \mathrm{DoF}$. Additionally, it was compared to a Multi-Layer Perceptron (MLP) which showed slightly but

M. Ortiz-Catalan is with the Dept. of Signals and System, Chalmers University of Technology, and the Centre of Orthopaedic Osseointegration, Dept. of Orthopaedics, Sahlgrenska University Hospital, Gothenburg, Sweden (e-mail: maxo@chalmers.se).

R. Brånemark is with the Centre of Orthopaedic Osseointegration, Dept. of Orthopaedics, Sahlgrenska University Hospital, Gothenburg, Sweden (email: rickard.branemark@orthop.gu.se).

B. Håkansson is with the Dept. of Signals and System, Chalmers University of Technology, Gothenburg, Sweden (e-mail: boh@chalmers.se).

This work was funded by VINNOVA (2010-00482) and Integrum AB. consistently better performance. Muceli et al. attributed this result to the MLP capabilities to handle non-linear relationships, and employed it for the prediction of hand kinematics including "hand close" as an additional movement [3]. As oppose to previous work predicting kinematics which requires additional hardware such as motion capture systems, and its designed for unilateral amputees [3], [4], the strategies presented in this study require only surface MES and therefore are equally valid for bilateral and unilateral amputees. Additionally, both "hand open and close" are included together with 4 wrist movements for a total of $3 \mathrm{DoF}$.

The aim of this study was to investigate different classifiers topologies capable of simultaneous prediction, and evaluated their performance in real-time in order to demonstrate the suitability of this approach. Until now, algorithms for simultaneous prediction have been evaluated using pre-recorded data only (offline) [1-4]. Since it has been shown that offline accuracy does not necessarily reflects real-time performance [5-9], the "motion test" introduced by Kuiken et al. [10] was used to provide realtime indicators.

Topologies such as One-Vs-All (OVA) and One-Vs-One (OVO) have been shown to outperform a single classifier predicting individual motions [8], [11], and although OVO yielded higher offline accuracies than OVA, it is inherently unable to predict simultaneous movements as the final output is computed by majority voting. On simultaneous classification, topologies with dedicated MLPs per DoF have also been argued to outperform a single classifier [4]. However, the results of our study show that this is not always the case, and stress the need for common data sets that allow proper inter-study comparisons.

This study was approved by the Swedish Regional Ethics Committee in Gothenburg (626-10, T688-12).

\section{Methods}

\section{A. Explored Classifier Topologies}

The following topologies were implemented in BioPatRec and evaluated using a MLP classifier [6].

- Single. This is the simplest and standard topology where all inputs feed the classifier and this is trained to discriminate all labels.

- Ago/Antagonist-Mixed (AAM). This topology assumes that the motions are paired in ago/antagonist movements (essentially a DoF). There are as many classifiers as DoF, and each 
Ortiz-Catalan, M., Brånemark, R., and Håkansson, B., Evaluation of Classifier Topologies for the Real-time Classification of Simultaneous Limb motions, in Proceedings of the $35^{\text {th }}$ Annual International Conference of the IEEE Engineering in Medicine and Biology Society. Osaka, Jul 3-7, 2013.

classifier is feed with the feature vectors of at least 3 classes; 2 of them are the antagonist motions related to the DoF; and the $3^{\text {rd }}$ is a mixed class combining all the other movements. A $4^{\text {th }}$ class is optional if the "rest" (no motion) information is available. The output vector contains the winner motion from each classifier.

- One-Vs-All (OVA). In this topology there are as many classifiers as classes. Each classifier is trained to discriminate between one class and a mixed class containing the others. The output vector contains the winner of each classifier.

The MLP had a sigmoid activation function and consisted of 2 hidden layers with the same hidden as input neurons. The training method was backpropagation with $\eta=0.1$ learning rate and $\alpha=0.1$ momentum. The training was stochastic by randomly supplying $70 \%$ of the available training sets per learning iteration. A maximum of 200 iterations was allowed for convergence.

\section{B. Data Acquisition and Processing}

Eight pairs of disposable Ag/AgCl electrodes in a bipolar configuration were placed equally distributed around the proximal third of the forearm; one distal and one proximal. The first pair (channel 1) was consistently placed along the extensor carpi ulnaris, and the rest following the pronation movement, or lateral direction. The bioelectric amplifier was an in-house design (MyoAmpF2F4-VGI8) with $66 \mathrm{~dB}$ gain, and embedded active filtering: 4th order high-pass filter at 20 $\mathrm{Hz}$; 2nd order low-pass filter at $400 \mathrm{~Hz}$; and, Notch filter at $50 \mathrm{~Hz}$. The signals were digitalized at $2 \mathrm{kHz}$ with 16-bits resolution.

The subjects were guided by the software (BioPatRec [6]) to execute and hold the motion during 3s, and relax during 3s between each contraction. Three repetitions of each movement result in 9s of raw MES information. The movements were hand open and close, wrist flexion and extension, and pro/supination, as well as all their possible combinations resulting in 26 motions plus "rest" (no motion).

We have previously found that under the presented recording method, $70 \%$ of the contraction time (cTp) normally eliminates periods of absent MES while conserving the isometric part of the contraction. This resulted in 121 time windows of $200 \mathrm{~ms}$ per movement (50 ms time increment), see [6] for further explanation on the signal processing and feature extraction.

Four time-domain signal features (mean absolute value, wave length, zero crossings, and slope sign changes) were extracted from each time window in order to from the feature vectors later used to feed the classifiers.

Six subjects participated in this study and their recording sessions, together with the relevant subject's information, are available in the BioPatRec's bioelectric signals repository under the folder "6mov8ChFUS_MLP_Topologies" [6].

\section{Training and Offline Evaluation}

The total of feature vectors was divided in $40 \%, 20 \%$, and $40 \%$ sets for training, validation, and testing, respectively. The feature vectors were randomly distributed in these sets before training. The testing set was only used to compute the offline accuracy once the training was completed. One hundred trainings were performed for each subject and topology.

Statistical significance was evaluated using the Wilcoxon Signed-Rank test, as it has been demonstrated to be appropriated for comparing different classifiers in common data sets [12]. Statistical significance was considered at $\mathrm{p}<0.05$, and values preceded by “ \pm ” represent the standard deviation.

\section{Real-time Evaluation}

The "Motion Test" was used for the real-time evaluation of the different classifier topologies [10]. Its implementation in BioPatRec and further description can be found in [6]. In summary, it consists on requesting the subject to execute the different motions in a randomized order while evaluating the following key performance indicators:

- Selection time. This is the time between the first prediction different than "rest", and the first correct prediction. It includes the time window length.

- Completion time. It uses the same trigger as the selection time but elapses on the $20^{\text {th }}$ correct prediction.

- Completion rate. This is the percentage of motions that achieved 20 correct predictions before timeout.

- Real-time accuracy. It is reported as the percentage of correct predictions over the total number of predictions that occurred between the first prediction different to zero and the $20^{\text {th }}$ correct prediction.

In order to avoid training bias, the topologies were tested in a different order for each subject. The subjects were allowed to familiarize themselves with the real-time prediction system during 5 minutes before performing each test. The motion test consisted of 2 trials where each movement was performed 3 times. The time out for motion completion was $10 \mathrm{~s}$, and data was available for prediction every $50 \mathrm{~ms}$.

\section{E. Implementation}

The topologies evaluated in this work were implemented in BioPatRec, an open source framework for the development of advanced prosthetic control strategies based on pattern recognition algorithms (PRAs) [6]. The implementation was done in a way that allows any PRA inside BioPatRec to be used automatically in any of the available topologies. This is by simply selecting the desired topology from a pop-menu.

BioPatRec's modular design allows a seamless implementation of algorithms on signal processing; feature selection and extraction; pattern recognition; and, real-time 
control. It includes all the necessary routines for the myoelectric control of virtual limbs, prosthetic devices, or game control; from data acquisition to real-time evaluations, including a virtual reality environment. Furthermore, it provides a freely available repository of bioelectric signals for algorithm's benchmarking on common data sets.

\section{RESULTS}

The graphical representation of the results is provided in box plots where a central mark represents the median value; the edges of the box are the 25th and 75th percentiles; the whiskers give the range of data values; and solid markers represent the mean values. Outliers greater than approximately $\pm 2.7 \sigma$ are not shown for clarity but considered in all calculations. A summary of the offline and real-time comparison between classifiers is presented in Table I.

TABLE I. COMPARISON BETWEEN MLP TOPOLOGIES

\begin{tabular}{|c|c|c|c|c|c|c|c|}
\hline & \multicolumn{3}{|c|}{ Offline } & \multicolumn{4}{|c|}{ Real Time } \\
\hline & $\begin{array}{c}\text { Testing* } \\
(\mu \mathrm{s})\end{array}$ & $\begin{array}{l}\text { Training } \\
\text { (s) }\end{array}$ & $\begin{array}{c}\text { Accuracy } \\
(\%)\end{array}$ & $\begin{array}{c}\text { Selection } \\
\text { Time (s) }\end{array}$ & $\begin{array}{c}\text { Completion } \\
\text { Time (s) }\end{array}$ & $\begin{array}{c}\text { Completion } \\
\text { Rate (\%) }\end{array}$ & $\begin{array}{c}\text { Accuracy } \\
(\%)\end{array}$ \\
\hline Single (S) & $98( \pm 1)$ & $32( \pm 21)$ & $93.4( \pm 2)$ & $0.85( \pm 0.2)$ & $2.68( \pm 0.4)$ & $90.6( \pm 7)$ & $52.4( \pm 6)$ \\
\hline OVA & $5.8 \times S( \pm 6)$ & $5.9 \times S( \pm 65)$ & $93.0( \pm 2)$ & $1.1 \times S( \pm 0.4)$ & $1.1 \times S( \pm 0.7)$ & $90.0( \pm 10)$ & $53.1( \pm 9)$ \\
\hline AAM & $3.2 \times S( \pm 6)$ & $2.5 \times S( \pm 34)$ & $93.2( \pm 3)$ & $0.9 \times S( \pm 0.3)$ & $0.9 \times S( \pm 0.6)$ & $89.9( \pm 11)$ & $56.1( \pm 8)$ \\
\hline
\end{tabular}

The average offline accuracy per classifier, and grouped by the number of combined movements, is shown in Fig. 1 . The selection and completion time from the motions test are shown in Fig. 2 and 3 respectively. The cumulative completion rate is displayed in Fig. 4, and finally, the realtime accuracy is shown in Fig. 5.

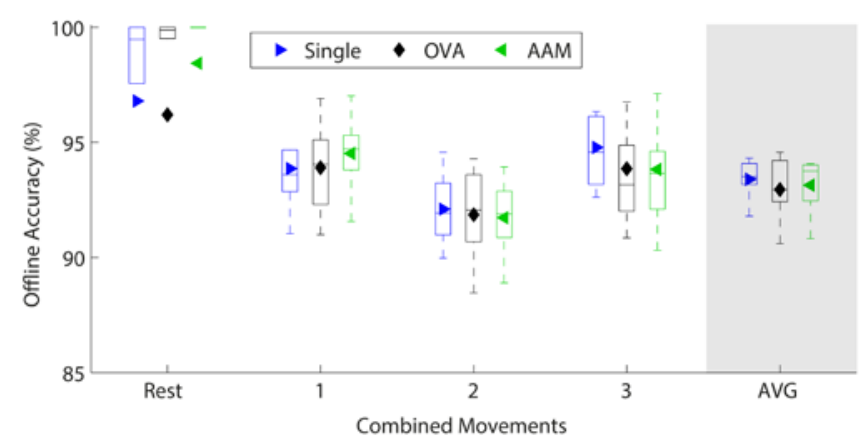

Figure 1. Offline accuracy grouped by the number of combined motions. "Rest" represent the "no motion" class. No statistical significance was found on the average for all movements.

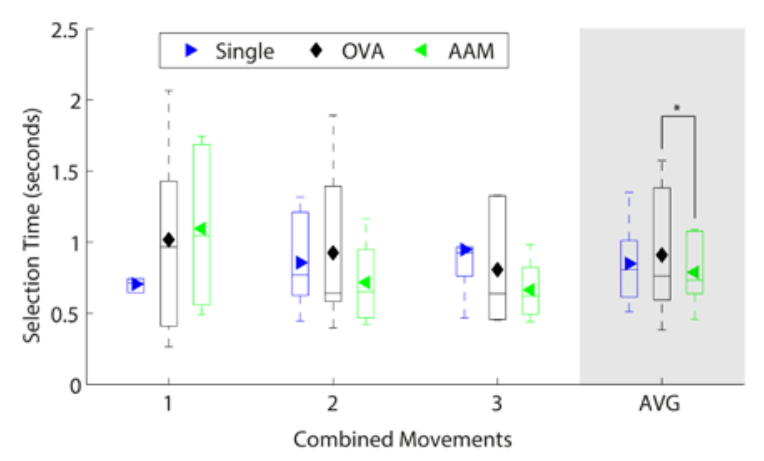

Figure 2. Selection time grouped by the combined motions. Statistical significance is shown by the marker " * ", and only for the average values.

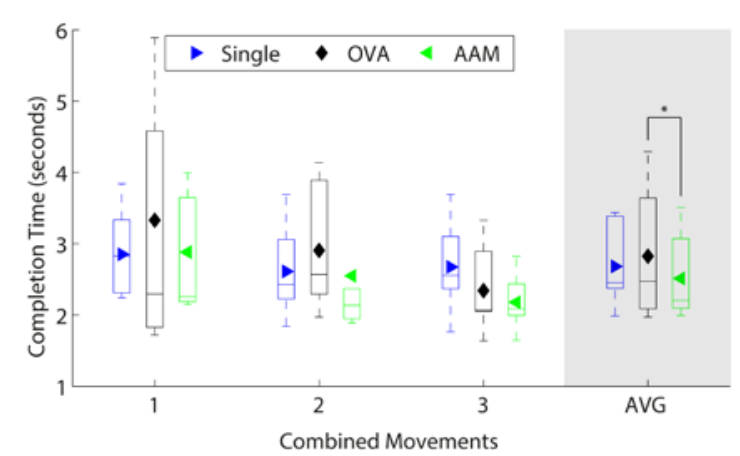

Figure 3. Completion time grouped by the combined motions. Statistical significance is shown by the marker " **, and only for the average values.

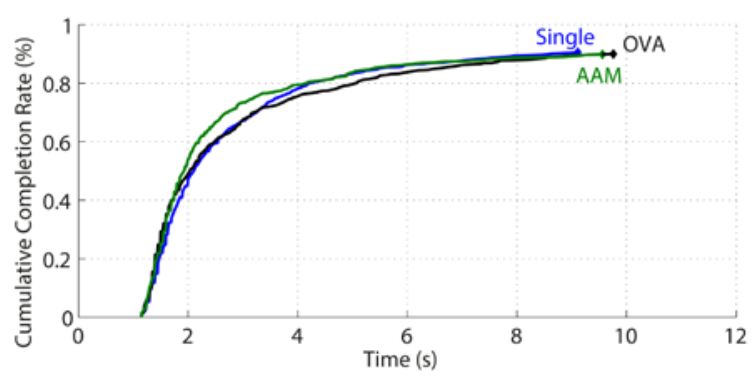

Figure 4. Cumulative completion rate plot by classifier.

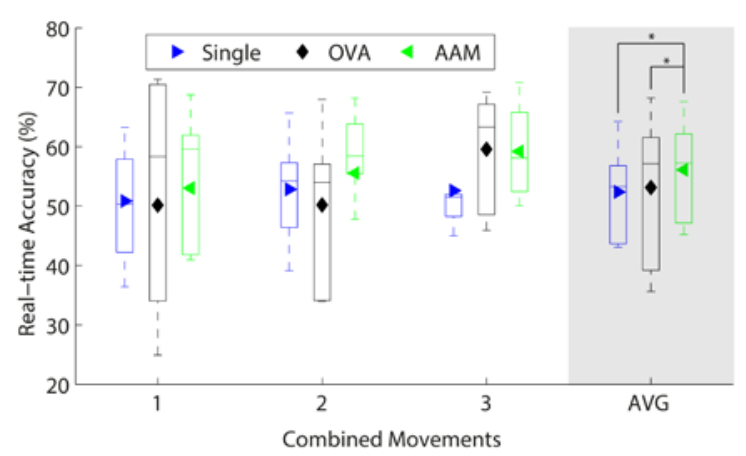

Figure 5. Real-time accuracy grouped by the combined motions. Statistical significance is shown by the marker “*”, and only for the average values.

\section{DISCUSSION}

The offline results show no perceivable difference in the classification accuracy, but as expected, the training and prediction times increased as more classifiers are used. The real-time results show a different picture than the apparent similarity observed offline. Interesting differences can be observed between the Single and AAM topologies when considering the number of combined motions. The single MLP was faster than AAM to initially predict individual movements, while slower to predict 3 movements combined (Fig. 2). This behavior was kept through the completion time (Fig. 3). However, since the real-time accuracy of the Single MLP was lower than AAM (Fig. 5), the difference in completion time became marginal for individual movements. This is because more misclassifications were made while trying to reach 20 correct predictions, thus increasing the completion time. 
Although the Single topology showed higher offline accuracies than AAM (Fig. 1), the real-time accuracy was found consistently opposite (Fig. 5). This is reflected in the lower average selection and completion times (Fig. 2 and 3), as well as in the cumulative completion rate, thus suggesting AAM as the best performing classifier (Fig. 4). It is worthy of notice, however, that these differences could still be considered as practically marginal if they are not appreciable by the user. The subjects reported hardly to differentiate between the Single and AAM topologies when testing their response on a virtual reality environment and prosthetic devices, Fig. 6. Furthermore, it could be argued that the introduction of control algorithms to mitigate spurious misclassification would make the difference between these topologies insignificant. Further studies are currently performed by our group to evaluate controllability using more close-to-reality tests.

A different scenario was found for OVA, as this was consistently inferior offline and real-time. Although the subjects did not perceived difference between Single and AAM, they all reported OVA as slightly detrimental to the system response. This finding would have been harder to conclude if only offline comparisons were performed, thus stressing the need of real-time evaluations.

Caution should be made on drawing conclusions from the presented absolute values in comparison with past and future studies. Time performance indicators strongly depend on the hardware used for computation. The variability introduced by the complexity of the task, including subject's motivation, experience, and skills, should not be overlooked. Therefore, and although all the presented classifiers show higher accuracy than in previous work, true comparison cannot be made due to all these variables. As a candidate solution for this problem, the topologies presented in this work, as well as the data generated, are freely available in BioPatRec [6]. This allows repeatability and further studies employing common data sets. Furthermore, it serves as ground platform for further research and collaborations.

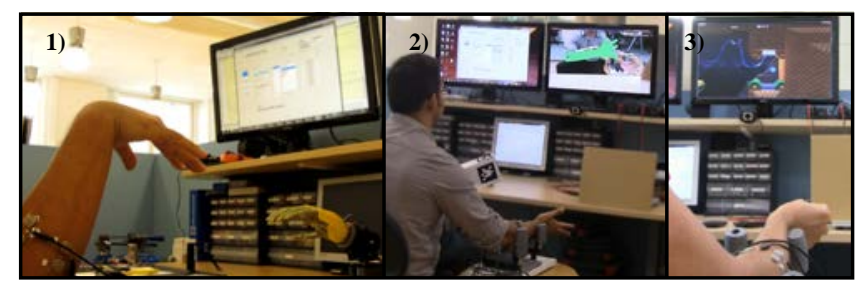

Figure 6. Simultaneous control of: 1) a multi-functional prosthetic device, 2) a virtual arm in an Augmented Reality, and 3) computer games. Videos of these demonstrations can be seen on-line in BioPatRec's projet site [13].

\section{CONCLUSION}

A more natural control of artificial limbs requires coordinated and simultaneous movements. The decoding of mixed myoelectric signals to predict such movements is an alternative to direct source isolation which is considerably challenging when using surface electrodes.
In this work, we demonstrated the feasibility of real-time simultaneous motion classification using surface electromyography only. More importantly, we provide a freely available and common development framework for further research. This has been made open source with the hope to accelerate, through the community contributions, the development of algorithms that will ultimately improve the patients' quality of life.

\section{ACKNOWLEDGMENT}

The authors thank N. Sander and M. B. Kristoffersen for their contribution to the virtual and augmented reality environments used together with BioPatRec. The authors also thank to all the participants in this study, who have generously contributed to the public repository.

\section{REFERENCES}

[1] D. Yatsenko, D. McDonnall, and K. S. Guillory, "Simultaneous, proportional, multi-axis prosthesis control using multichannel surface EMG,” in Conf Proc IEEE Eng Med Biol Soc, 2007, pp. 6134-7.

[2] N. Jiang, K. B. Englehart, and P. Parker, "Extracting simultaneous and proportional neural control information for multiple-DOF prostheses from the surface electromyographic signal.," IEEE Trans Biomed Eng, vol. 56, no. 4, pp. 1070-80, Apr. 2009.

[3] S. Muceli and D. Farina, "Simultaneous and Proportional Estimation of Hand Kinematics From EMG During Mirrored Movements at Multiple Degrees of Freedom.," IEEE Trans Neural Syst Rehabil Eng, vol. 20, no. 3, pp. 371-8, May 2012.

[4] N. Jiang, J. L. Vest-Nielsen, S. Muceli, and D. Farina, "EMG-based simultaneous and proportional estimation of wrist/hand dynamics in uni-Lateral trans-radial amputees,” J Neuroengineering Rehabil, vol. 9, no. 1, p. 42, 2012.

[5] B. A. Lock, K. Englehart, and B. Hudgins, "Real-time myoelectric control in a virtual environment to relate usability vs. accuracy," in Proceedings of the 2005 MyoElectric Controls/Powered Prosthetics Symposium, New Brunswick, Aug 17-19, 2005.

[6] M. Ortiz-Catalan, R. Brånemark, and B. Håkansson, "BioPatRec: A modular research platform for the control of artificial limbs based on pattern recognition algorithms," Source Code for Biology and Medicine, 8:11.

[7] G. Li, A. E. Schultz, and T. Kuiken, "Quantifying pattern recognitionbased myoelectric control of multifunctional transradial prostheses.," IEEE Trans Neural Syst Rehabil Eng, vol. 18, no. 2, pp. 185-92, 2010.

[8] E. J. Scheme, K. B. Englehart, and B. S. Hudgins, "Selective classification for improved robustness of myoelectric control under nonideal conditions.," IEEE Trans Biomed Eng, vol. 58, no. 6, pp. 1698-705, 2011.

[9] L. Hargrove, Y. Losier, B. A. Lock, K. Englehart, and B. Hudgins, “A real-time pattern recognition based myoelectric control usability study implemented in a virtual environment," in Proc. 29th Annu. Int. Conf. IEEE EMBS, Lyon, Aug 22-26, 2007, pp. 4842-5.

[10] T. A. Kuiken, G. Li, B. A. Lock, R. D. Lipschutz, L. A. Miller, K. A. Stubblefield, and K. B. Englehart, "Targeted muscle reinnervation for real-time myoelectric control of multifunction artificial arms," JAMA: the journal of the American Medical Association, vol. 301, no. 6, pp. 619-628, 2009.

[11]L. J. Hargrove, E. J. Scheme, K. B. Englehart, and B. S. Hudgins, "Multiple binary classifications via linear discriminant analysis for improved controllability of a powered prosthesis.," IEEE Trans Neural Syst Rehabil Eng, vol. 18, no. 1, pp. 49-57, 2010.

[12]J. Demsar, "Statistical comparisons of classifiers over multiple data sets," The Journal of Machine Learning Research, vol. 7, pp. 1-30, 2006.

[13]M. Ortiz-Catalan, "BioPatRec [http://code.google.com/p/biopatrec],” Accessed Jan, 2012. 